\title{
Exploring the Utilization of Non-Invasive Methods for Assessing Health Status of Patients with Chronic Respiratory Inflammation within the United Arab Emirates
}

\author{
Sadiya Al Menhali ${ }^{1}$ and Fatme Al Anouti ${ }^{*}$ \\ ${ }^{1}$ Environment, Health \& Safety Section, School Services Division, Abu Dhabi Education Council, Abu Dhabi, UAE \\ 2Zayed University, Natural Science and Public Health Department, Abu Dhabi, UAE \\ "Corresponding author: Fatme Al Anouti, Assistant Professor, Zayed University, Natural Science and Public Health Department, Abu Dhabi, UAE, Tel: \\ 971-503-019-807; E-mail: Fatme.AlAnouti@zu.ac.ae
}

Received date: March 31 2014, Accepted date: April 18, 2014, Published date: April 26, 2014

Copyright: (C) 2014 Fatme AA, et al. This is an open-access article distributed under the terms of the Creative Commons Attribution License, which permits unrestricted use, distribution, and reproduction in any medium, provided the original author and source are credited.

\begin{abstract}
Asthma is defined as the chronic inflammation of the large airways that is characterized by reversible airflow obstruction and airway hyper-responsiveness. On the other hand, Chronic Obstructive Pulmonary Disease (COPD) is a chronic inflammation that is marked by progressive and irreversible airflow obstruction. Both conditions are manifested by symptoms of wheezing, coughing, and shortness of breath. Similarity in symptoms between these two conditions could lead to misdiagnosis and mistreatment. Although inflammation is present in both conditions, there are some marked differences. The main difference is that inflammation in asthma is an IgE mediated and involves the release of histamine while it is absent in the case of COPD. There is now a new trend for measuring the content of exhaled nitric oxide as a marker for assessing airway inflammation and especially for asthma diagnosis and monitoring. This study aimed at utilizing biomarkers to differentiate between the different types of respiratory inflammations. In this study, 23 patients with asthma, 20 with allergic rhinitis, 10 with COPD, and 17 healthy controls were recruited into the study at a local hospital clinic and assessed based on the level of their exhaled nitric oxide (eNO), serum specific lgE antibodies, and relative blood eosinophilia count. The results demonstrated that the many asthmatics $(74 \%)$ had high eNO levels, while only $25 \%$ of patients with allergic rhinitis, $10 \%$ of those with COPD, and $6 \%$ of the healthy individuals similarly had elevated eNO levels. The results confirmed the fact that exhaled nitric oxide could be an effective parameter for differentiating asthma from other allergic diseases (e.g. allergic rhinitis) and other respiratory inflammations (COPD).
\end{abstract}

Keywords: Asthma; Allergic diseases; Inflammation

\section{Introduction}

Allergic diseases (asthma, rhinitis, and eczema/dermatitis) could be atopic and non-atopic. Atopy is the genetic contribution or the tendency for the development of allergic diseases [1]. It is characterized by the genetic predisposition for IgE production in response to exposure to inhalant or food allergens. The risk of a child developing allergic diseases is $20 \%$ if both parents are not affected, but the risk increases with a single defective parent to be $25 \%-30 \%$ and $50-75 \%$ if both is allergic [2].

Both environmental and genetic factors contribute to the development of an allergic phenotype. Evidence for genetic influence in the etiology of allergy was first established through twin studies. In 1971, Edfors- lubs studied 7000 Swedish twin pairs and the results for monozygotic versus dizygotic were as the following: asthma $19 \%$ vs. $4.9 \%$, rhinitis $21.4 \%$ vs. $13.6 \%$, and eczema $15.4 \%$ vs. $4.5 \%$, respectively [3]. As there are many molecules involved in the inflammatory response, scientists were able to identify more than 100 genes causing asthma and other allergic diseases [4]. The genome wide search approach has established an association between allergic manifestations and markers on 14 pairs of chromosomes such as 1,2 , $3,5,6,7,9,11,12,13,14,16,17,19$ [5]. The expression of certain Human Leukocyte Antigen (HLA) haplotype corresponds to the exhibition of a certain clinical phenotype. For example, asthmatics have the haplotype HLA B7, SC31, DR2 while allergic rhinitis (AR) patients have the haplotype HLA B8, SC01, DR3 [6]. Environmental factors such as air borne allergens, air pollutants, and microbial antigens are also considered to be a risk factor for the development of allergic diseases. Thus, allergic phenotypes are the result of complex interactions between genetics and the environment $[7,8]$.

Air pollutants can contribute to the etiology or severity of allergic diseases. Environmental or air pollutants are classified into primary or secondary, based on their composition whether they are gases or particulate matter (PM), and location (indoors or outdoors) [9]. Primary agents are those directly released into the atmosphere such as $\mathrm{SO}_{2}, \mathrm{NO}_{\mathrm{x}}$, and $\mathrm{CO}_{2}$ while those resulting from interaction with other compounds such as ozone are called secondary pollutants. The main source for outdoor air pollutants production is motor vehicles, industry, and energy generating facilities and that for indoor pollutants is fuel or wood burning stoves. Emissions from car exhaust are made of volatile organic compounds, $\mathrm{PM}$, and poisonous gases such as $\mathrm{CO}, \mathrm{CO}_{2}, \mathrm{SO}_{2}, \mathrm{NO}_{\mathrm{x}} \cdot \mathrm{NO}_{2}$ is a toxic gas forming the photochemical smog with its ability to also form acid rain. $\mathrm{NO}_{2}$ concentrations are elevated in the center of cities and low in the rural areas. On the other hand, ozone levels are high away from the city center and low at the center because of its ability to react with NO forming NO2. People with asthma or COPD get sensitized to lower doses of $\mathrm{NO}_{\mathrm{x}}$ resulting in increased bronchial hyper-reactivity [9]. 


\section{Diagnosis of Allergy}

The diagnosis of the previous allergic conditions requires many tests. Skin tests especially skin prick tests are widely used in clinical practice. An aqueous solution of common inhaled allergens such as HDM, pollen, cat dander, and dog hair, occupational allergens (ammonium persulphate, platinum salts, antibiotics, and latex), and food allergens are used. A positive control (histamine) and negative control (diluents) are also used. In general practice, a drop of each common inhalant allergens as well as the positive and negative controls are placed on the skin of forearm pricked by a sterile lancet needle specific for each allergen and left for 15 minutes. A positive result is confirmed by a skin wheal of $>2 \mathrm{~mm}$ greater than that seen by the diluents. Bronchial and nasal provocation tests can be also performed if skin prick and RAST tests were negative. A certain concentration of histamine or metacholine is administered to the patient that causes $20 \%$ drop in $\mathrm{FEV}_{1}$. Nasal smears are undertaken to estimate the number of eosinophil in the nasal discharges. A cotton bud is placed in each nostril and the mucosa is scraped with a film rolling movement and then the cotton bud are gently spread into a microscopic slide and stained and the cells are counted. Positive result is determined if more than $10 \%$ of the stained cells are eosinophils. The other test is radio allergosorbent (RAST) or serum allergen specific IgE levels. The allergens are covalently bounded to cellulose. If the patient's serum contains IgE antibodies, it will react to its specific allergen complex. Non-specific IgE are washed off and then specific anti IgE conjugate is added to the IgE bound allergen with a fluorescent marker. The fluorescence of each complex is measured by the machine and printed out. Any reading above $0.35 \mathrm{ku} / \mathrm{l}$ is considered as positive. Bronchodilator challenge test using spirometery is also used to check the presence of reversible airway obstruction. The patient should take a deep breath applying a nose clip, and then exhale the air for six seconds as fast and hard as he can through the mouthpiece that is connected to the machine by a tube, finally the machine measures the total amount of air exhaled (FVC) and how much air exhaled in one second (FEV1) and the results are printed out Asthmatic have $\mathrm{FEV}_{1} / \mathrm{FVC}$ value of $0.7 \mathrm{~L}$ or $70 \%$ before the test, but after doing it they show an increase in $\mathrm{FEV}_{1}$ by at least $12 \%$ and at least $200 \mathrm{~mL}$ in FVC pointing to reversible airflow obstruction.

\section{COPD}

\section{Definition, classification, and diagnosis:}

COPD is defined as a "progressive and irreversible airflow obstruction of the peripheral airways". COPD has three main components including chronic obstructive broncholitis with fibrosis and small airway blockage, emphysema with the enlargment of airspaces and damaged lung parenchyma, and chronic bronchitis that is mucus hyper-secretion. Smoking, air pollution (poisonous gases, diesel exhust, ozone, and $\mathrm{PM}$ ), and occupational exposure to dust are the main causes for the development of COPD. Smoking accounts for $>95 \%$ of all cases as being a risk factor Symptoms of COPD include productive cough, sputum, and most importantly shortness of breath. GOLD (Global Intiative for Obstructive Lung Disease) classifies the severity of COPD into 4 stages according to spirometery.All four stages have a ratio of $\mathrm{FEV}_{1} / \mathrm{FVC}$ around $70 \%$ or $0.7 \mathrm{~L}$ while the exact value is dependent on age, height, weight, sex, and ethnicity. Stage I (mild) is charactarized by $\mathrm{FEV}_{1}>80 \%$,stage II (moderate) FEV1 is between $50 \%-80 \%$, stage III (severe) $\mathrm{FEV}_{1}$ within $30 \%-50 \%$, and stage VI (very severe) FEV $1<30 \%$ or $<50 \%$ plus chronic respiratory failure.
Spirometery as well as mediacl history have been used in the diagnosis of COPD. Confirm the diagnosis of COPD involves looking into its irrversibility component. Performing bronchodilator challenge test in COPD patients $\left(\mathrm{FEV}_{1} / \mathrm{FVC} 0.7 \mathrm{~L}\right.$ or $\left.70 \%\right)$ shows no increase by $12 \%$ in $\mathrm{FEV}_{1}$ or $200 \mathrm{~mL}$ in FVC.Its prevalence is very high in smokers and exsmokers, people over 40 years of age, and more specifically males.

\section{Differences between asthma and COPD:}

Although inflammation is present in both asthma and COPD, there are some markeddifferences in inflammatory cells and mediators, limitation of airflow, parenchymal integrity, (BHR), response to antiinflammatory therapy, and onset of symptoms Barnes et al. [1]. In asthma, the main cells recruited are $\mathrm{TH} 2(\mathrm{CD} 4+)$ lymphocytes, eosinophils, and mast cells while in COPD Tc1 (CD8+) lymphocytes, neutrophils, and macrophages are involved in the inflammatory response. The main mediator released in asthma is histamine that is absent in COPD. TH2 lymphocytes release IL-4 that causes the production of IgE from B-lympocytes while Tc1 produce INF- $\gamma$ inhibiting IgE synthesis. Airflow limitation is largely reversible in asthma, but it is largely irreversible in COPD. The lung parenchyma in asthma is intact as compared to COPD, which is badly destructed. BHR is significant feature of asthma with its being variable (small) component of COPD. Asthmatics respond very quickley to steriod traetment in comparison to the presence of steriod resistance in COPD patients. Asthmatics have almost normal lung function with treatment compared to everyday symptoms of COPD even though the use of bronchodilaros and smoking cessation [1]. Onset of asthma is very early during childhood while COPD develops in smokers and exsmokers in their mid 40s [1]. Histopathology studies showed that the inflammation in COPD affects the peripheral airways (bronchioles) and lung parenchyma whereas the whole airway without the involvment of parenchyma is inflammed in asthma.

\section{Nitric Oxide}

\section{Discovery of importance}

$\mathrm{NO}$ is the most widely studied exhaled marker of airway inflammation). Interest in nitrate has been old enough when Alfred Nobel invented the dynamite in 1863 . He eventually died of angina pectoris from the work on it. In 1916, dietary studies showed that the body is internally producing nitrate with the source being L-arginine amino acid. In 1980, research within the cardiovascular field not the pulmonary area showed the significance of NO. They suggested that the stimulation of endothelium by acetylcholine caused the release of a substance that easily diffuses and targets smooth muscle cells of the arteries causing their vasodilatation in isolated arterial lungs. Removal of that substance stopped such relaxation. They named it EDRF, endothelium derived relaxing factor. In 1986, EDRF was recognized as NO because of their similar properties.

\section{Physical and chemical properties}

Physical and chemical properties of $\mathrm{NO}$ are, as the following. At room temperature, it is colorless gas and in the absence of $\mathrm{O}_{2}$, it dissolves in water. It is made of one nitrogen and 1 oxygen atoms so it is a small molecule. It has a short half-life depending on its concentration and physio-chemical environment. It has an odd number of electrons. This property makes it a free radical that can diffuse easily from its site of formation being highly reactive with many other atoms and free radicals in biological systems. It reacts with 
$\mathrm{O}_{2}$ forming nitrate and nitrite. In the presence of superoxide anion $\left(\mathrm{O}_{2}^{-}\right)$, NO can react with it forming $\left(\mathrm{OONO}^{-}\right)$, a toxic anion causing lipid peroxidation impairing the cell membrane resulting in cell death and DNA destruction. It also binds to thiols producing S-nitrosothiols such as S-nitrosocysteine and S-nitrogluththione. They act as NO donors when needed. NO have high affinity for the ferrous $\left(\mathrm{Fe}^{2+}\right)$ part of the haemoproteins such as haemoglobin, myoglobin, cytochrome $\mathrm{C}$ and soluble guanylatecyclase leading to the production of nitrosyl substances.

\section{Significance in biological systems}

NO is a critical molecule as it has a pivotal role in neurotransmission, regulationof vasodilation, pulmonary host defence by acting as bacteriostatic or bactericidal, cell mediated immunity, ciliary beat frequency, mucus secretion, plasma exudation, and as a marker of inflammation. $\mathrm{NO}$ is released from inhibitory nonadergenic non-cholinergic nerves causing the bronchodilator effect in human airways. Smooth muscles are present in vascular endothelium and the bronchi so NO regulates pulmonary arterial vasoconstriction. The use of NOS inhibitors proved to reduce the vasodilatory response in pulmonary arteries in vivo and in animal studies. Cells that produce NO are fibroblasts, endothelial and epithelial cells, keratinocytes, chondrocytes, monocytes/macrophages, APCs, natural killer (NK) cells, eosinophils, and mast cells. Low levels of NO are essential for normal body functioning, but there is disease conditions that alter NO level to be high. A study on normal mice showed that epithelial cells express minor levels of NOS 2 that is responsible for NO release in exhaled air. With the proliferation of inflammatory cytokines, the induced NOS isoform is expressed causing the increased eNO in asthmatics. In asthma, the increased expression of NOS 2 in airway epithelial cells is linked to elevated transcription process through transcription factor (STAT 1) and nuclear transcription factor (NF $\kappa B$ ) withthe increased presence of L-arginine. The major cells contributing to the increased NO levels expressing iNOS are epithelial cells, macrophages, and eosinophilsand those origins are relevant to asthma.Astudy measured the variability of eNO among asthmatics compared to normal subjects. They found that eNO was elevated in asthmatics compared to normal controls. It was higher in moderatesevere asthmatics than mild and normal people. Their finding suggested that eNO was not significantly different in mild asthmatics than normal controls. They also showed that there is a decreased expression of IFN- $\gamma$ in moderate-severe asthmatics. This is because increased eNO level inhibits Th1 cytokine expression. A study by Taylor et al. showed that cloned murine Th1 cells activated by antigens were able to express iNOS and caused NO production. Further NO production inhibited Th1 cell proliferation and so IL- 2 and IFN- $\gamma$ release. The level of eNO is altered within the respiratory tract. The level of eNO from the URT is (200-1000 p.p.b. (parts per billion)), from the sinuses it is (1000-30000 p.p.b.), and the LRT it is very low about (1-9 p.p.b.). The paranasal sinuses have high eNO level as there is an evidence for continuous expression of NOS 2 .

\section{Exhaled nitric oxide measurement}

eNO measurement requires a single breath exhalation technique. In 1997, eNO was found to be inversely proportional to expiratory flow rate in what is called flow dependence. Therefore, high flow rate reduces eNO while low flow rate increases eNO. There was a need to standardize the flow rate so the European Respiratory Society (ERS) and the American Thoracic Society (ATS) identified a certain flow rate for eNO measurements and that is $50 \mathrm{ml} / \mathrm{s}$. As discussed previously
NO is released from the URT and LRT and detecting lung inflammation should not include the nasal NO. eNO measurement ensures the exclusion of nasal NO through making the subject inhale to total lung capacity without a nose clip followed by exhalation at a constant flow rate of $50 \mathrm{~mL} / \mathrm{s}$ against resistance with positive mouth pressure that should range between $5-20 \mathrm{~cm} \mathrm{H} 2 \mathrm{O}$ closing the velum and so no NO interrupts the measurement. eNO was found to be significantly correlated with other markers of inflammation such as airway hyper-responsiveness, sputum and blood eosinophil, and total and specific IgE.

There are many factors affecting eNO level. Age was proved to affect the eNO level. eNO was seen to increase in children with age. Also, sex affects eNO level. A recent study conducted showed that eNO levels are 25\% higher in males than females (males: $15.3 \mathrm{ppb}$ [95\% C.I.: 14.3-16.3] vs. females: 11.6 ppb [95\% C.I.: 11.0-12.4]; $\mathrm{p}<0.0001)$. In this study, atopic individuals were found to have increased eNO level by $60 \%$. Another study showed that normal values for adult exhaled nitric oxide are 2.6- 28.8 p.p.b or 2.6- $28.8 \mathrm{nl} / \mathrm{l}$ for males and 1.6-21.5 p.p.b for females. Thus, differences between males and females account also for normal eNO values. Breath-holding, low exhalation flow rate, high ambient $\mathrm{NO}$, nasal NO, nitrate rich food, inhaled or ingested L-arginine, exercise are factors causing the increased eNO. On the other hand, high exhalation flow rate, sputum induction, bronchial provocation tests, spirometery, NOS inhibitors, use of corticosteroids, oral leukotriene antagonists, and smoking and passive smoking are related to reduced eNO level. Taking into accountthe previous factors, Aerocrinecompany in Sweden designed many NO analyzers such as NIOX NO monitoring system and NIOX MINO hand held device using the ERS/ATS guidelines. They designed those machines as the patient inhales NO free air through a disposable mouth piece with flow dependency on $50 \mathrm{ml} / \mathrm{s}$ preventing contamination from nasal NO and ambient NO. eNO level is defined as a 3-second NO plateau at the end of exhalation and the patients can ensure a valid result by getting visible (screen) and audible (continuous sound) feedback.eNO should be measured prior to exercise, sputum induction, bronchial provocation tests, and spirometery. Some respiratory diseases may also increase or decrease eNO level while others show variable changes.

There are many advantages for eNO measurement. It has high reproducibility. Khartinovo and colleagues showed high reproducibility using the NIOX NO monitoring system with a standard deviation of $2.1 \mathrm{ppb}$. High reproducibility of eNO in both children and adults allows the medical technician to repeat the measurement twice with more reliable results instead of three exhalations as compared to spirometer. Another study by Alving et al. showed high success rate of $(\geq 84 \%)$ using the NIOX and NIOX MINO machines in clinical (with practitioner help) and home set (without). This demonstrates easy use of the machine by patients especially the hand held NIOX MINO. eNO has been used in the diagnosis of asthma. It predicts future asthma or asthma exacerbations especially in patients with subclinical symptoms such as normal function tests, negative bronchodilator tests, and low sputum ECP (eosinophilic cationic protein) concentrations having high eNOlevel. Harkins et al. looked into the use of eNO as a monitoring tool for predicting future asthma exacerbations. They studied 22 moderatesever asthmatics and demonstrated that after 2 weeks of initial appointment in a routine clinic visit some of them had exacerbations with a high mean eNO $(29.67 \mathrm{ppb} \pm 14.48)$ compared to those without exacerbation, who had mean eNO value of (12.92 ppb \pm 5.17$)$ $(\mathrm{p}=0.002)$. eNO was found in the study to be statistically significant 
$(\mathrm{p}=0.031)$ compared to FEV1 $(\mathrm{p}=0.56)$ in the prediction of future asthma exacerbations. Because eNO is a non-invasive and cost effective method, it can be used to monitor a large populat. eNO has been used in measuring the effectiveness of anti-inflammatory drug. In addition, eNO is very sensitize to corticosteroid treatment as it decreases after $6 \mathrm{hrs}$ of a single use of this treatment or 2-3 days even reaching the maximum level after 2-4 weeks of therapy. Shome et al. study concluded that eNO is declining in moderate-severe asthmatics with steroid therapy and by 4 weeks it was not significantly different from mild asthmatics or healthy controls. In a study conducted by Silkoff et al. 62 patients ( 33 children and 32 adults) with uncontrolled asthma were assessed to observe the effect of steroid treatment on the level of eNO. eNO were recorded at visit 1 and visit 2 . The results for both age groups showed high significant decreased in eNO from visit 1 to visit 2 with a mean standard deviation percentage change in eNO levels of $-50.5 \% \pm 31.41 \%(\mathrm{P}<0.001)$. Reductions in steroid intake result in an increase in eNO level by $40 \%$ and $100 \%$ after 2 and 4 weeks respective. High sensitivity of eNO to steroid treatment helps in adjusting the dose of corticosteroids because high doses harm the patients. In some cases of persistent asthma symptoms, eNO remains high despite the use of corticosteroids as being a marker indicating steroid resistance. Also, eNOlevel respond quickly to any changes in the airways before other parameters change such as lung function tests and sputum eosinophil being a good sign for loss of control. The increase in eNO is accompanied by reduced lung function tests and increased asthma related symptoms leading to exacerbations. Sputum induction is believed to be invasive and difficult to perform in children. Lung function and bronchial provocation tests have limitations because they are not directly related to airway inflammation and can be easily affected by bronchodilators (Khartinovo and Barnes, 2001). eNO is directly related to the inflammatory response as it is released by many inflammatory cells. Both of them take a significant amount of time to change and they are not able to assess the effect of various steroid doses. Using NIOX analyzers does not require that much staff training as it operates automatically.

Because asthma is characterized by eosinophilic inflammation, it can be detected with elevated eNO levels. In stable COPD, the inflammation is mainly neutrophilic suggesting lower levels of eNO than smokers and nonsmokers with asthma not different from normal subjects being less likely to respond to steroids (Khartinovo and Barnes, 2001). Chronic cigarette smoking decreases eNO. This is because increased NO in cigarettes down regulate NOS and oxidants in the smoke inactivates NO release. This may responsible for the decreased eNO in COPD patients. Maziak et al. showed that eNO is higher in unstable COPD patients than current smokers with COPD, ex-smokers with COPD, and smokers with chronic bronchitis without airflow obstruction $(12.7 \mathrm{ppb} \pm 1.5 \mathrm{ppb})(\mathrm{p}<0.01$. eNO were higher in smokers with COPD compared to smokers with chronic bronchitis $(4.3 \mathrm{ppb} \pm 0.5 \mathrm{ppb}$ vs. $2.5 \mathrm{ppb} \pm 0.5)(\mathrm{p}<0.05)$ and even higher in exsmokers with COPD (6.3 ppb $\pm 0.6 \mathrm{ppb})(\mathrm{p}<0.01)$. eNO also was high in COPD patients under steroid treatment (most are severely ill) in relation to steroid naïve COPD patients $(8.2 \mathrm{ppb} \pm 1.2 \mathrm{ppb}$ vs. $5 \mathrm{ppb} \pm$ $0.4 \mathrm{ppb})(\mathrm{p}<0.05)$. There was not a significant difference between COPD steroid naïve and COPD steroid treated in terms of eNO indicating steroid resistance in COPD. In patients with severe disease, the increase in eNO far much exceeds its decrease due to smoking. Severe COPD cases in the previous study $\left(\mathrm{FEV}_{1}<35 \%\right)$ showed high eNO level compared to stable COPD. Thus, it can be used to assess disease activity in COPD and monitor response to steroid treatment.
This is because some COPD patients with concomitant asthma exhibit high eNO levels.

\section{Objectives}

To compare the significance of eNO and other available markers (serum specific IgE, and relative blood eosinophil) in achieving a clear diagnostic criteria between inflammatory respiratory diseases aiming at better management and control.

To establish a reliable objective marker for diagnosis and monitoring of asthma. To determine the contribution of allergy in the etiology of asthma.

\section{Methods}

Participants: 76 subjects were enrolled in the study. They were 23 asthmatics, 20 with allergic rhinitis, 10 with COPD, and 17 healthy individuals. The age range was 7-60 years both males and females. They were randomly selected from different ethnic backgrounds. Patients were attending the outpatient clinics at a local hospital within Abu Dhabi (especially those in the ENT, pediatric, and medical clinics). They were referred to the allergy clinic to be informed more about the study. Asthmatics were pre-diagnosed according to the GINA guidelines. Allergic rhinitis patients were already seen by ENT consultants or by an allergist. COPD patients were also pre-diagnosed by chest physicians. The final group was the healthy controls, who were hospital staff or patients who were visiting the clinic for routine annual medical checkup.

A case study sheet was prepared for each patient pertaining to personal and demographic information, family history of disease, symptoms occurrence, provoking factors, and social factors. Informed consent was obtained from all participants and also an ethical approval was granted from the hospital's IRB. Patients with chronic debilitating diseases such as diabetes mellitus, liver diseases, cancer and those taking immune-suppression therapy were excluded from the study. All patients were assessed for eNO level, relative blood eosinophil count, and RAST tests.

\section{Exhalednitric oxide (eNO) level}

The patient's eNO level was measured by using the NIOX MINO ${ }^{\circ}$ portable machine (Aerocrine, Sweden). The patient should empty his lungs and then inhale from the machine until full lung capacity and exhales against a constant positive counter pressure of $10-20 \mathrm{~cm} \mathrm{H}_{2} \mathrm{O}$ to ensure a flow rate of $50 \mathrm{ml} / \mathrm{s}$ for 10 s preventing nasal contamination as well. The machine displays a reading determining the content of NO in the exhaled air. Normal values based on the previous flow rate are 1.6-28.8 p.p.b for both adult males and females and the upper limit for children aged $4-17$ is 25 p.p.b.

\section{Specific IgE test (RAST)}

Specific IgE antibodies in patient's serum could be assessed using the Immuno CAP Flourenzymatic immunoassay technique with the use of UNI CAP $100^{\ni}$ machine. The machine with the allergens is provided by (Pharmacia Diagnostis, Uppsala, Sweden) (Ownby, 1993). The allergens are covalently bounded to a cellulose solid phase called ImmunoCAPs. Patient's serum, controls, the reagents, and the ImmunoCAPs are loaded into their specific locations. The machine transmits a specific quantity of serum and the controls to the ImmunoCAPs initiating the first incubation. If the serum includes 
specific IgE, it will combine with its specific allergen in the ImmunoCAPs. After washing to remove any unbounded IgE, specific anti-IgE conjugate (with a fluorescent detector) is added to the ImmunoCAPs starting the second incubation. Finally, a stop solution is transferred and the fluorescence of each ImmunoCAP is assessed by the machine. Any reading above $0.35 \mathrm{ku} / \mathrm{l}$ is considered as positive (Ownby, 1993).

\section{Relative blood eosinophil count}

WBC could be analyzed using Automated Hematology Analyzer machine (XE-2100) which gives complete blood count mainly WBC (NEUT, LYMPH, MONO, BAS, EOS), RBC, PLT, HGB. The required blood sample volume in the tube is $1 \mathrm{~mL}$ or more. More than $4 \%$ eosinophil was reported as abnormal.

\section{Data Analysis}

All data was analyzed using SPSS version 21 (Chicago, USA. the analysis using this software revealed the correlations between eNO and other factors (age and blood eosinophil).

\section{Results}

The results indicated that there was no significant difference in mean values between healthy non-smoker males $(\mathrm{N}=4)$ and healthy non-smoker females $(\mathrm{N}=13)$ in the level of eNO (Figures 1 and 2). Results from Table 1 showed that $74 \%$ of asthmatics had high eNO compared to those with COPD, allergic rhinitis, and healthy groups, who had $10 \%, 25 \%$, and $6 \%$, respectively. Percentage of asthmatics having high blood eosinophil was $52 \%$, COPD was $0 \%$, AR was $17.6 \%$, and healthy was $30 \%$. RAST test positivity to one or more inhalant allergens showed no difference between asthmatics (91\%) and allergic rhinitis patients $(75 \%)$ in contrast to the case of COPD $(30 \%)$ and healthy (29.4\%). Figure 2 compares the three parameters between the four groups. Results also revealed that $\mathrm{eNO}$ and age were negatively associated, [-0.26], $\mathrm{p}<0.05$. Also, eNO and eosinophil were positively associated [0.46], $\mathrm{p}<0.01$. The level of eNO was significantly high among asthmatics compared to the other groups. This significance had an $\mathrm{F}$ value of $20.02, \mathrm{p}<0.01$. The eNO levels demonstrated that there was a difference between groups.

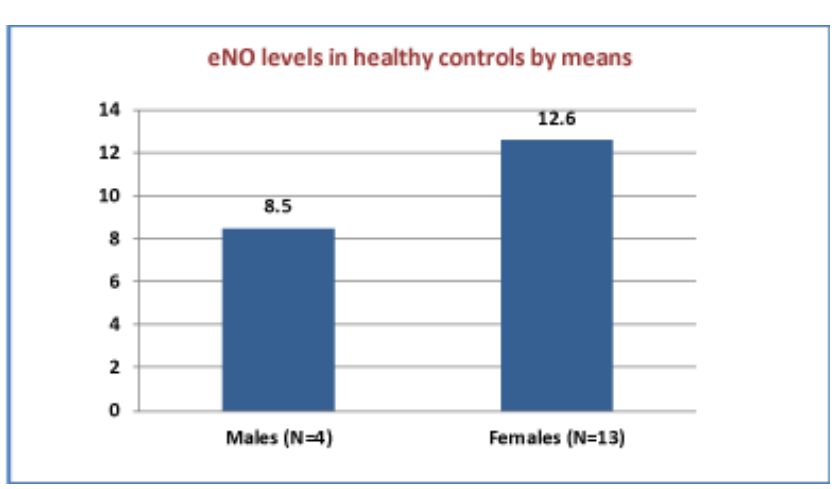

Figure 1: eNO levels in healthy controls by means.

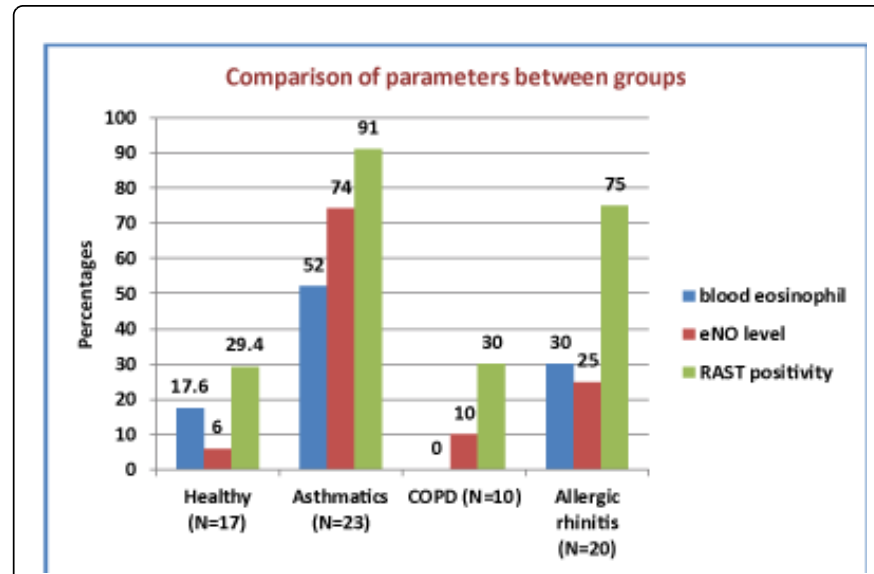

Figure 2: Comparison of parameters between groups.

\begin{tabular}{|l|l|l|l|l|l|l|}
\hline Clinical state & \multicolumn{2}{|l|}{$\begin{array}{l}\text { \% with high Exhaled } \\
\text { nitric oxide } \\
\text { (eNO) }\end{array}$} & $\begin{array}{l}\text { \% with } \\
\text { Raised blood } \\
\text { eosinophilia }\end{array}$ & \multicolumn{2}{|l|}{\begin{tabular}{l}
\multicolumn{2}{l|}{ with } \\
positive RAST
\end{tabular}} \\
\hline & No & $\%$ & No & $\%$ & No & $\%$ \\
\hline Healthy (N=17) & 1 & 6 & 3 & 17.6 & 5 & 29.4 \\
\hline $\begin{array}{l}\text { Asthmatics } \\
(\mathrm{N}=23)\end{array}$ & 17 & 74 & 12 & 52 & 21 & 91 \\
\hline COPD (N=10) & 1 & 10 & 0 & 0 & 3 & 30 \\
\hline $\begin{array}{l}\text { Allergic rhinitis } \\
(\mathrm{N}=20)\end{array}$ & 5 & 25 & 6 & 30 & 15 & 75 \\
\hline
\end{tabular}

Table 1: The results indicated that there was no significant difference in mean values between healthy non-smoker males $(\mathrm{N}=4)$ and healthy non-smoker females $(\mathrm{N}=13)$ in the level of eNO (Figure 1 and 2).

\section{Discussion}

Asthma is defined as a chronic airway inflammation characterized by reversible airflow obstruction, yet most physicians still rely on subjective measures (as frequency of attacks of shortness of breathes) in the diagnosis and monitoring of asthma. These measures are mostly inconsistent and do not reflect the real inflammatory pathology. In this preliminary study, an attempt was made to find an objective parameter to be used as a reliable marker for asthma diagnosis and monitoring.

Three clinical groups of patients; those with asthma; those with allergic rhinitis and those with COPD as well as a healthy group were investigated. Three laboratory tests were used, the exhaled Nitric oxide level, the percentage blood eosinophilia and the allergic sensitization tests. The results of this preliminary study clearly showed that exhaled nitric oxide level is higher in asthmatic patients compared to healthy individual and it is relatively higher than other clinical groups with respiratory inflammation. Blood eosinophilia does not show such significant difference among different groups. This is because blood eosinophilia is a general parameter for allergy and is not specific for respiratory allergy while eNO level is specific for airway inflammation. Moreover, blood eosinophilia is affected by many other factors such as intestinal infestation, eczema etc. while eNO is not. Most studies have 
demonstrated that NO level in the exhaled air corresponds to the extent of eosinophilic airway inflammation (Alving et al., 2006). eNO shows better correlation with sputum eosinophilia rather than with blood eosinophilia.

We found that in patients with COPD the levels of eNO were not very high, this is because inflammation in COPD is characterized by neutrophils infiltration rather than eosinophils. These patients are known to have poor response to steroids therapy, in contrast to asthmatic patients with high eNO levels, who are good responders.

Regarding the degree of allergic sensitization as measured by RAST test, we found that asthmatic patients as well as allergic rhinitis patients showed a higher degree of positive allergic sensitization to one or more inhalant allergens as compared by healthy individual. However, RAST test is not specific for asthma as it can be positive in other allergic disorders (e.g. allergic rhinitis, atopic eczema, food allergy etc.). Moreover, there were few asthmatics, which showed negative RAST test. In this study, we found that around $87 \%$ of our asthmatics had high positive sensitivity to dust mite allergens. This shows that allergy to inhalant allergens in UAE contributes greatly to the etiology of asthma and that allergic asthma is more common in Abu Dhabi population than non-allergic (intrinsic) asthma. This may be due to the type of housing in Abu Dhabi, which is closed system ventilation with door to door carpet fitting, in addition to, the high humidity all over the year due to its location near the Arabian Gulf. These factors increase indoor dust mite concentrations, which tend to accumulate mainly on bed covers, carpets, furniture, and children's soft toys and cause sensitization of susceptible persons. Moreover, there is an enormous increase in construction projects especially in Abu Dhabi and Dubai, hence increasing air pollution and favoring the risk of sensitization. It is known that as humidity increases reaching $75 \%$ and under room temperature of $25^{\circ} \mathrm{C}$, droppings of mites become suspended in the air and when inhaled by genetically predisposed persons cause asthma or allergic rhinitis symptoms. Most of patients are treated by immunotherapy to boost their immune system making it less responsiveness to dust when encountered over and over. Another important finding from this study is that some allergic rhinitis patients had high eNO levels without any symptoms of asthma. This is a threatening indication for future development of asthma and the physicians should be aware of it in order to prevent progression. Therefore, the allergist should describe intranasal as well as inhaled or oral corticosteroids for treating both conditions together.

Thus, it is apparent that eNO can be used as a biomarker for assessing the extent of airway inflammation and help in the diagnosis and monitoring of asthma even before the onset of clinical symptoms of airway obstruction. It can be used to differentiate asthma from other respiratory disorders especially COPD, which does not show very high levels of eNO.

Our results demonstrated that the high eNO level in asthmatic patients dropped markedly and rapidly in response to steroid therapy and thus, could be used to monitor the response of patient to treatment and guide its dose and duration. It is far superior to the traditional methods that have been used for monitoring asthma such as spirometery and sputum induction, which as discussed previously in the introduction, require longer time to show the changes (Scientific Backgrounder, 2003). Furthermore, spirometry can be sometimes normal (during the quite reversible state) in spite of, ongoing airway inflammation while eNO level will remain high even in sub clinical state as it reflects exactly the state of airway pathology. Corticosteroids treatment has a direct effect on switching off the cascade of inflammatory process and thus will lower eNO level immediately. In COPD, inflammation is not suppressed by corticosteroids as it is produced by other mechanisms. The biomarker eNO is an applicable measurement to all age groups and easy to perform especially among children.

Levels of eNO were negatively correlated with age, $\mathrm{p}<0.05$. This correlation is not expected as children grow up, their eNO levels increase. It can be assumed that the small sample size in this study as only few children were included compared to the adult group may not precisely reflect the age effect. If larger sample was studied, it would be more representative. The effect of gender on eNO levels was not significantly different between healthy non-smoker males and females though literature proved the opposite. One study by Taylor et al. has shown a significant gender related difference in eNO levels even after controlling the other factors such as smoking, and atopy. This can be also due to the small sample size studied. Further investigations with larger study samples are needed to provide concrete evidence. Also longitudinal studies with follow up after therapy would be more informative. However, this preliminary study had demonstrated the applicability of eNO measurement as a monitoring tool for airway inflammation in clinical practice in the UAE.

\section{References}

1. American Academy of Allergy, Asthma \& Immunology (2013) Allergic conditions: Atopy Retrieved April 15.

2. Toda M, Ono J (2002)Genomics and proteomics of allergic disease. Immunology106: 1-10.

3. Sheikh A (2002) Itch, sneeze and wheeze: The genetics of atopic allergy. J the Royal Society of Med95: 14-17.

4. Zhang J, Paré P, Sandford A (2008) recent advances in asthma genetics. Respir Res 9: 4

5. Wang D (2005) Risk factors of allergic rhinitis: Genetic or environmental?T herapeutics and Clinical Risk Management1: 115-123.

6. Bousquet J, Cauwenberge P, Khaltaev N (2001) Allergic rhinitis and its impact on asthma. The Journal of Allergy and Clinical Immunology 108.

7. Dávila L, Mullol J, Bartra J, CuvilloA, Ferrer M, et al. (2007). Effect of pollutants upon patients with respiratory allergies. J Investig Allergolclin Immunol 17: 9-20.

8. World Allergy Organization(2014) Combined allergic rhinitis and asthma syndrome. Allergic diseases resources center.RetrievedMay 18, 2014.

9. Mahr TA, Malka J, Spahn JD (2013) Inflammometry in pediatric asthma: a review of fractional exhaled nitric oxide in clinical practice. Allergy Asthma Proc. May-Jun 34:210-219. 